\title{
Protection against canine parvovirus type 2 infection in puppies by colostrum-derived antibodies
}

\author{
Hanna Mila ${ }^{1,2,3}$, Aurélien Grellet ${ }^{3}$, Costantina Desario ${ }^{4}$, Alexandre Feugier ${ }^{3}$, Nicola Decaro ${ }^{4}$, \\ Canio Buonavoglia ${ }^{4}$ and Sylvie Chastant-Maillard ${ }^{1,2 *}$ \\ ${ }^{1}$ Université de Toulouse, INP, ENVT, UMR 1225, IHAP, F-31076 Toulouse France \\ ${ }^{2}$ INRA, UMR1225, IHAP, F-31076 Toulouse, France \\ ${ }^{3}$ Royal Canin, 650 Avenue de la Petite Camargue, Aimargues, France \\ ${ }^{4}$ Department of Animal Health and Well-Being, Faculty of Veterinary Medicine, Bari University, Bari, Italy
}

(Received 23 January 2014 - Final revision received 18 August 2014 - Accepted 2 September 2014)

Journal of Nutritional Science (2014), vol. 3, e54, page 1 of 4

doi: $10.1017 /$ jns. 2014.57

Abstract

During the first weeks of life puppies remain protected against canine parvovirus type 2 (CPV2) infection thanks to maternally derived antibodies (MDA) absorbed with colostrum after birth. The objective of the present study was to present the variability in CPV2-specific passive immune transfer and its consequences in puppies naturally exposed to the parvovirus. Seventy-nine puppies from one breeding kennel were included in the study at birth and followed until $56 \mathrm{~d}$ of age. Once per week the MDA titre for CPV2 specific antibodies was determined in blood. Viral excretion was also evaluated on a rectal swab by CPV2 PCR assay and puppies were weighed to determine growth rate. At $2 \mathrm{~d}$ of age, thirty-four out of seventy-nine puppies (43\%) had MDA $\leq 1: 160$ (designed group A) and forty-five puppies $(57 \%)$ had greater MDA titres (designed group B). The level of absorbed maternal antibodies was shown to be associated with breed size and growth rate during the first $48 \mathrm{~h}$ of life. The MDA level declined with age in all cases; however, the proportion of puppies with the antibody level considered as protective against CPV2 infection was significantly higher in group B compared with A from day 2 until 42. Among all puppies surviving until $56 \mathrm{~d}$ of age, sixty-seven out of seventy $(95.7 \%)$ underwent CPV2 infection. However, puppies from group A excreted CPV2 significantly earlier than puppies from group B. The present study demonstrates the link between passive immune transfer, in terms of level of specific MDA absorbed, and length of the protection period against parvovirus infection in weaning puppies.

Key words: Puppies: Canine parvovirus: Colostrum: Maternally derived antibodies

The prevalence of canine parvovirus type 2 (CPV2) in diarrheic puppies varies from $64 \%$ in North America and $70 \%$ in Europe $^{(1,2)}$. CPV2 is a ubiquitous enteropathogen that is responsible for outbreaks of acute gastroenteritis, with a high mortality rate ${ }^{(3)}$. During the first weeks of life, maternally derived antibodies (MDA) provide the only specific systemic protection against CPV2 in puppies. Only $10 \%$ of circulating CPV2 antibodies in the neonate are from transplacental ori$\operatorname{gin}^{(4)}$. The vast majority is transferred from the dam to puppies through colostral ingestion during the first hours of life. Systemic CPV2 MDA titre decreases with age ${ }^{(4-6)}$. When the serological titre has fallen under 1:80 (haemagglutination inhibition (HI)), the systemic MDA level seems to be no longer protective against $\mathrm{CPV} 2^{(4,7)}$. To date, the variability in maternally derived protection against CPV2 and its consequences in puppies have been studied exclusively under experimental conditions and only on weaned puppies or puppies deprived from maternal milk. Since canine colostrum and milk have been proven to provide significant amounts of CPV2 antibodies ${ }^{(6)}$, these lactogenic MDA could potentially interfere with CPV2 intestinal replication either by coating the enterocytes or trapping the faecal CPV2 particles, preventing their multiplication

Abbreviations: CPV2, canine parvovirus type 2; HI, haemagglutination inhibition; MDA, maternally derived antibodies

* Corresponding author: Professor S. Chastant-Maillard, email s.chastant@envt.fr 
in the mucosa. Moreover, since viral challenge induces a more rapid decrease in circulating CPV2 antibodies, ${ }^{(8)}$ MDA kinetics may differ depending on environmental infective pressure.

In the present study, we focused on CPV2 MDA in puppies maintained under natural conditions, housed in a breeding kennel with natural CPV2 circulation. The aim of the work was to analyse the variability and kinetics of systemic maternally derived CPV2 antibodies under these field conditions and to evaluate factors influencing MDA. The link between initial MDA level, viral shedding and growth performance was also studied.

\section{Materials and methods}

The study protocol was reviewed and approved by the Royal Canin Internal Ethics Committee.

\section{Animals}

The experiment was conducted in a commercial breeding kennel over a 4 months period (March-June 2012). Seventy-nine puppies from various breeds (twenty-six litters, ranging from one to eight puppies per litter alive at day 2 ; day $0=$ whelping) were included and followed from birth until $56 \mathrm{~d}$ of age. Breeds whose adult weight was less than $25 \mathrm{~kg}$ were considered small breeds and dogs with a greater adult weight large breed dogs. All puppies were housed with their dam in heated whelping boxes from birth to $56 \mathrm{~d}$. Puppies were allowed to suckle freely. Lactating bitches and their puppies were fed ad libitum with the same diet, a dry expanded complete diet balanced for growing dogs (Starter, Royal Canin).

Blood (1 ml per puppy) was collected from the jugular vein at days 2 and 7 and every week until day 56. Samples were immediately centrifuged $(3000 \mathrm{~g}, 15 \mathrm{~min})$ and serum separated. Rectal swabs were performed at day 17 and every week until day 52. Sera and rectal swabs were stored for 4 months at $-20^{\circ} \mathrm{C}$ until assayed.

\section{Canine parvovirus type 2 antibodies assay}

Titres of antibodies directed against CPV2 were evaluated on serum by $\mathrm{HI}$ test as previously described ${ }^{(7)}$. Tests were performed at $+4^{\circ} \mathrm{C}$ using ten haemagglutinating units of CPV2 antigen and $1 \%$ pig erythrocytes. All samples from one puppy (from one to nine samples) were tested on one plate with a maximum of ten plates performed at one time. Twofold dilutions in PBS of each serum sample starting from 1:10 were tested and the $\mathrm{HI}$ titre was the highest serum dilution completely inhibiting viral haemagglutination. Titres below 1:80 were considered as non-protective against infection ${ }^{(4)}$. Seroconversion was defined as a minimum 4-fold increase of HI titre. After a seroconversion episode (indicating a viral contamination), puppies were considered as no longer protected by MDA.

\section{Canine parvovirus type 2 faecal excretion}

A homogenate $(10 \%)$ of the faecal sample was prepared in PBS (pH 7.2) and centrifuged at $1500 \mathrm{~g}$ for $15 \mathrm{~min}$. The viral DNA was extracted from prepared supernatant by boiling the sample $(10 \mathrm{~min})$ and subsequently chilling on ice. To reduce inhibition of DNA polymerase, samples were diluted 1:10 with distilled water. No more than ten extractions were performed at one time. CPV2 real-time PCR assay with the TaqMan probe was conducted on faecal samples as described by Decaro et al. ${ }^{(9)}$ with ovine herpesvirus 2 DNA as internal control. A dilution of standard DNA in a CPV-negative faecal suspension was performed (serial $\log 10$ dilutions) and tested to determine the detectability and the linearity of the assay. The following thermal protocol was used: iTaq DNA polymerase was activated at $95^{\circ} \mathrm{C}$ for $10 \mathrm{~min}$ followed by forty cycles consisting of denaturation at $95^{\circ} \mathrm{C}$ for $15 \mathrm{~s}$, subsequently primer was annealed at $52^{\circ} \mathrm{C}$ for $30 \mathrm{~s}$ and the process was extended at $60^{\circ} \mathrm{C}$ for $1 \mathrm{~min}$.

\section{Growth}

Puppies were weighed at birth, $48 \mathrm{~h}$ and every week until $56 \mathrm{~d}$ of age using a calibrated analytical scale in $1 \mathrm{~g}$ increments (Fisher Scientific International Inc.). Subsequently, growth rate $(\%)$ over the first $2 \mathrm{~d}$ of life was calculated ((weight at $2 \mathrm{~d}$ - weight at birth)/weight at birth $\times 100)$, together with growth rate between 21 and $56 \mathrm{~d}$ of life ((weight at $56 \mathrm{~d}-$ weight at $21 \mathrm{~d}) /$ weight at $21 \mathrm{~d} \times 100)$.

\section{Statistical analysis}

Statistical analyses were performed using Tanagra ${ }^{\circledR}$ freeware (Tanagra 1.4, Lyon, France). All datasets were tested for normality by the Shapiro-Wilk test. As data were not normally distributed, they were presented as medians and range. A twosided Mann-Whitney $U$ test or a Kruskal-Wallis test was used according to the number of groups considered. The level of statistical significance was set at $P<0.05$ for all analyses.

\section{Results}

Variability in canine parvovirus type 2-specific passive immune transfer

At $2 \mathrm{~d}$ of age, MDA titres displayed large variability between puppies, titres ranging from $1: 10$ to $1: 1280(\log 10=1-3 \cdot 1$; Fig. 1). At that time (day 2), thirty-four out of seventy-nine puppies $(43 \%)$ had HI titre $\leq 1: 160(\log 10 \leq 2 \cdot 2$; group A), among which thirteen (38\% of total population) had not reached the $\mathrm{HI}$ titre $1: 80(\log 10=1.9)$, considered as the minimal protection against CPV2 infection. Only forty-five of seventy-nine animals (57\%) had HI titres $>1: 160$ (group B) with seven puppies at $\mathrm{HI} \geq 1: 1280$. Mortality rate between 2 and $56 \mathrm{~d}$ of age was significantly higher in puppies from group A than group B $(9 / 34 ; 26 \% v .3 / 45 ; 7 \% ; P=0.022)$.

\section{Factors influencing canine parvovirus type 2-specific passive immune transfer}

The breed size and growth rate between birth and $48 \mathrm{~h}$ of life were associated with CPV2 specific antibody transfer from bitch colostrum to puppies. Large breed puppies had higher 


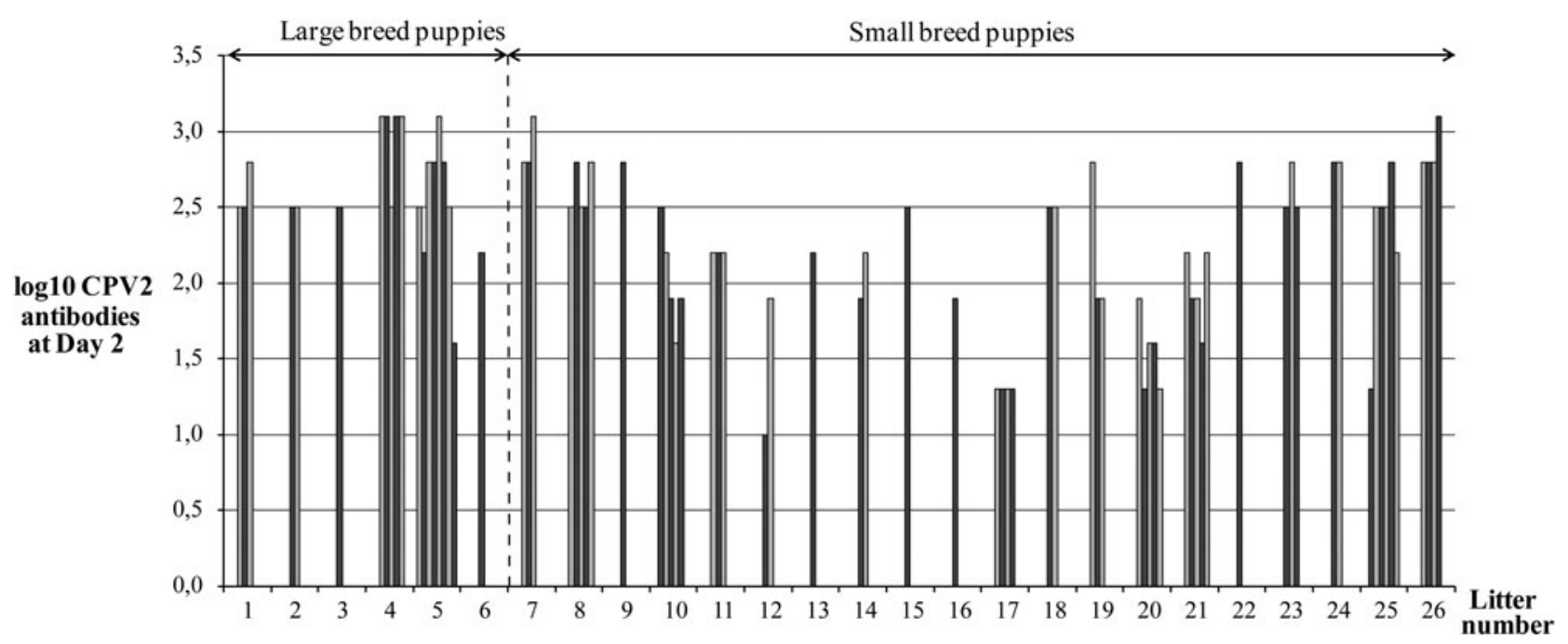

Fig. 1. Variability in maternally derived antibody titres against canine parvovirus type 2 at $2 \mathrm{~d}$ of age ( $n$ 79). Each bar represents one puppy, each group of bars represents one litter ( $x$-axis), and puppies are divided in small and large breeds (arrows).

MDA titres at day 2 compared with small breed puppies (median HI titre: 1:320 (range: 1:40-1:1280) v. 1:160 (1:101:1280); $P=0.003)$. Puppies which lost weight during the first $48 \mathrm{~h}$ of life had lesser MDA titres at day 2 than puppies which gained weight (HI titre: 1:120 (1:10-1:1280) v. 1:320 (1:40-1:1280); $P<0 \cdot 001)$.

\section{Maternally derived antibody level and canine parvovirus type} 2 infection

Kinetics of canine parvovirus type 2 maternally derived antibodies. The titres of MDA progressively declined with age in both groups. The proportion of puppies with MDA protection against CPV2 infection in group B was significantly higher from day 2 until 42 than in group A. At day 56 none of the 67 surviving puppies displayed MDA titre $\geq 1: 80$ (Table 1).

Sixty-three out of the sixty-seven puppies still alive at the end of the experiment underwent a seroconversion, with sixtytwo of them reaching an HI titre of 1:1280. The four puppies which did not seroconvert belonged to group B. Median age at seroconversion was $49 \mathrm{~d}(28-56)$ in puppies from group $\mathrm{A}$ and $56 \mathrm{~d}(35-56)$ in group $\mathrm{B}(P<0 \cdot 001)$. Among thirteen puppies with MDA titre at day $2<1: 80$, the first seroconversion appeared at day 42. Half-life of systemic MDA was $13.4 \mathrm{~d}$.

Viral excretion. Among seventy puppies still alive at $17 \mathrm{~d}$ of age, sixty-seven $(96 \%)$ displayed a significant viral excretion
( $\geq 1000$ copies) at some point during the study period. Puppies from group A excreted CPV2 at significantly earlier age compared with puppies from group B (day 38 (17-52) $v$. day 45 (17-52); $P=0 \cdot 011)$.

At the time of the first significant viral excretion, fifty-two puppies had HI titres less than 1:80, fourteen puppies had HI titres of 1:80 or 1:160 and only three puppies had HI titres $>1: 160$.

Growth. Growth rates between 21 and $56 \mathrm{~d}$ of age were not significantly different between groups A and B (65\% (53-83\%) v. $62 \%(28-79 \%) ; P=0 \cdot 11)$.

\section{Discussion}

MDA are crucial for the protection of puppies against CPV2 infection since puppies are nearly agammaglobulinemic at birth. Canine neonates acquire systemic antibodies via colostral ingestion within the first hours of life before gut closure ${ }^{(10)}$. In the present study, at $2 \mathrm{~d}$ of age, MDA titres displayed large variability between puppies, with titres ranging from 1:10 to 1:1280. The variability in MDA level could be due to unequal colostrum ingestion from maternal or puppy's origin. In the present study, we noted a relationship between the early growth rate and the absorption of specific CPV2 MDA. Both reflect colostrum intake as this secretion plays not only an immune, but also a nutritional role. Systematic weighing of puppies could therefore be performed by breeders in

Table 1. Proportion of puppies protected from CPV2 infection $(H I \geq 1: 80)$ depending on MDA level at $2 \mathrm{~d}$ of age

\begin{tabular}{|c|c|c|c|c|c|c|c|c|c|}
\hline & \multicolumn{9}{|c|}{ Age of puppies (weeks) } \\
\hline & 2 & 7 & 14 & 21 & 28 & 35 & 42 & 49 & 56 \\
\hline Group A & $21 / 34(62)$ & $14 / 30(47)$ & $5 / 26(19)$ & $0 / 26(0)$ & $0 / 25(0)$ & $0 / 25(0)$ & $0 / 25(0)$ & $0 / 25(0)$ & $0 / 25(0)$ \\
\hline Group B & $45 / 45(100)$ & 44/44 (100) & 41/44 (93) & $34 / 44(77)$ & 24/42 (57) & $10 / 44(23)$ & 9/44 (20) & $2 / 44(5)$ & $0 / 43(0)$ \\
\hline$P$-value for each period of time & $<0.001$ & $<0.001$ & $<0.001$ & $<0.001$ & $<0.001$ & 0.011 & 0.021 & 0.531 & - \\
\hline
\end{tabular}

$n_{\mathrm{i}} / n=$ number of puppies protected in the category considered/total number of puppies in the category (\%). 
order to control for correct passive immune transfer and energy intake at the very early stages of life.

After the first $24 \mathrm{~h}$ of life, MDA are no longer absorbed and they decline with age ${ }^{(4,6)}$. Pollock \& Carmichael $^{(4)}$ observed a half-life for CPV2 MDA of $9.7 \mathrm{~d}$, with puppies reaching seronegative levels between 10 and 14 weeks of age. Gooding \& Robinson $^{(5)}$ observed the HI titres $<1: 10$ after day 49 . In the present study, half-life was slightly longer at $13.4 \mathrm{~d}$. From the observation of Macartney et al. ${ }^{(8)}$, who described an acceleration in the decline of blood CPV2 titres after viral challenge, one could expect a more rapid MDA decrease in the present study, which was conducted under a high CPV2 environmental pressure. In this situation, systemic MDA may be recruited to limit the multiplication of CPV2 virus, thereby leading to an earlier entry into a susceptibility period for viral infection. Nevertheless, in our conditions of natural infection, this hypothesis was not confirmed.

The early consumption of a sufficient quantity of maternal colostrum to maximise passive immune transfer appears to increase the length of the protective period. Indeed, the proportion of protected puppies was higher in the group with the higher MDA level (group B) until $42 \mathrm{~d}$ of age. The study demonstrates thus the importance of optimal colostrum intake in puppies in order to induce a longer immunoprotection during the pediatric period.

The large variation in the CPV2 susceptibility period between puppies observed in the present study underlines that a routine vaccination protocol should be adapted not only to the breeding kennel epidemiologic situation, but also to a puppy's individual needs. Although the early vaccination appears controversial since MDA may interfere with CPV vaccination, decreasing the vaccines response $\mathrm{e}^{(4,11,12)}$, recently a high antigen titre vaccine administrated as early as 4 weeks of age was demonstrated effective in the reduction of the CPV2 susceptibility window ${ }^{(13)}$.

Over the study period, nearly all puppies (96\%) underwent viral infection and seroconversion. Viral infection appeared in the vast majority of puppies when $\mathrm{HI}$ titres were lower or equivalent to $1: 80$ (90\% of the infected puppies with $\mathrm{HI} \leq$ $1: 80)$, as described for experimental viral challenges ${ }^{(4,7)}$. In puppies with a MDA level lower than protective against CPV2 infection at birth, seroconversion appeared for the first time only at day 42. Further work would be necessary to assay pathogenic viral loads (by haemagglutination test) in parallel with global viral load (as obtained by PCR), together with coproantibodies ${ }^{(11)}$, to verify the importance of lactogenic MDA before and during natural CPV2 infection episodes.

Conclusions. Based on systemic MDA, optimal passive immune transfer lengthens the protection period against CPV2 infection. Breeders should be encouraged to pay attention to early suckling within the first $12 \mathrm{~h}$ after birth. Systematic weighing at early age, evaluating indirectly the passive immune transfer, could indicate puppies at risk and allow adaptation of the vaccination protocol to individual needs. Nevertheless, the potential role of local MDA, as provided by milk, to limit viral replication and its consequences on morbidity and mortality merits further investigation.

\section{Acknowledgements}

We would like to thank the owner of the kennel for his contribution to this work and Mr Adrian Watson for the English revision of the manuscript. The present study was partially funded by Royal Canin SAS (Aimargues, France) (grant no. R3789 1/02/2012). H. M., A. G. and S. C. M. contributed to the study design. H. M. carried out the study. H. M. and C. D. carried out the laboratory analyses and administrated the data. H. M., A. G. and A. F. performed statistical analyses. H. M., A. G and S. C. M. drafted the manuscript and N. D. and C. B. improved it. The authors declare that there is no conflict of interest.

\section{References}

1. Kapil S, Cooper E, Lamm C, et al. (2007) Canine parvovirus types 2c and 2b circulating in North American dogs in 2006 and 2007. J Clin Microbiol 45, 4044-4047.

2. Decaro N, Desario C, Amorisco F, et al. (1997) Detection of a canine parvovirus type $2 \mathrm{c}$ with a non-coding mutation and its implications for molecular characterisation. Vet J Lond Engl 196, 555-557.

3. Glickman LT, Domanski LM, Patronek GJ, et al. (1985) Breed-related risk factors for canine parvovirus enteritis. $J \mathrm{Am}$ Vet Med Assoc 187, 589-594.

4. Pollock RV \& Carmichael LE (1982) Maternally derived immunity to canine parvovirus infection: transfer, decline, and interference with vaccination. I Am Vet Med Assoc 180, 37-42.

5. Gooding GE \& Robinson WF (1982) Maternal antibody, vaccination and reproductive failure in dogs with parvovirus infection. Aust Vet J 59, 170-174.

6. Decaro N, Desario C, Campolo M, et al. (2004) Evaluation of lactogenic immunity to canine parvovirus in pups. New Microbiol 27, 375-379

7. Decaro N, Campolo M, Desario C, et al. (2005) Maternally-derived antibodies in pups and protection from canine parvovirus infection. Biol J Int Assoc Biol Stand 33, 261-267.

8. Macartney L, Thompson H, McCandlish IA, et al. (1988) Canine parvovirus: interaction between passive immunity and virulent challenge. Vet Rec. 122, 573-576.

9. Decaro N, Elia G, Martella V, et al. (2005) A real-time PCR assay for rapid detection and quantitation of canine parvovirus type 2 in the feces of dogs. Vet Microbiol 105, 19-28.

10. Chastant-Maillard S, Freyburger L, Marcheteau E, et al. (2012) Timing of the intestinal barrier closure in puppies. Reprod Domest Anim 47, 190-193.

11. Rice JB, Winters KA, Krakowka S, et al. (1982) Comparison of systemic and local immunity in dogs with canine parvovirus gastroenteritis. Infect Immun 38, 1003-1009.

12. Burtonboy S, Charlier P, Hertoghs J, et al. (1991) Performance of high titre attenuated canine parvovirus vaccine in pups with maternally derived antibody. Vet $\operatorname{Rec} 128,377-381$.

13. De Cramer KGM, Stylianides E \& van Vuuren M (2011) Efficacy of vaccination at 4 and 6 weeks in the control of canine parvovirus. Vet Microbiol 149, 126-132. 